\title{
METAPLASIA ÓSEA ENDOMETRIAL
}

\section{REPORTE DE UN CASO Y REVISIÓN DE LITERATURA}

\author{
Giovani Castro Cuenca MD*, Paula Rodríguez MD**
}

\section{Resumen}

Mujer de 37 años que acudió al hospital para estudio de infertilidad con una ecografía pélvica que mostró una imagen hiperrefringente dentro de la cavidad endometrial similar a un dispositivo intrauterino. Estaba asintomática y no tenía antecedentes de importancia excepto un aborto espontáneo trece años antes. Se practicó histeroscopia encontrando múltiples fragmentos óseos, el mayor de $\mathbf{2} \mathbf{~ c m}$ de longitud, los cuales se retiraron utilizando pinzas de agarre sin complicaciones. El reporte de patología informó metaplasia ósea endometrial.

Palabras clave: metaplasia ósea, histeroscopia, infertilidad.

\section{OSSEUS METAPLASIA OF THE ENDOMETRIUM}

\section{CASE REPORT AND LITERATURE REVIEW}

\begin{abstract}
Thirty-seven-year-old woman admitted to the hospital for infertility work-up. Pelvic ultrasound showed a hyperechogenic image within the endometrial cavity mimicking an intrauterine device. Patient was asymptomatic and had no important antecedents except for a spontaneous abortion thirteen years before. Hysteroscopy revealed multiple osseous fragments, the biggest greater than $\mathbf{2} \mathbf{~ c m}$ in length. Fragments were removed with grasping clamps with no complications. Pathologist report established the diagnosis of osseous metaplasia of the endometrium.
\end{abstract}

Key words: osseous metaplasia, hysteroscopy, infertility.

\section{Introducción}

La metaplasia ósea endometrial es un raro padecimiento con una incidencia aproximada de 3:10.000 casos, ${ }^{1}$ que a menudo se asocia con infertilidad. En la literatura se encuentran reportados dos clases de pa- decimientos: la verdadera metaplasia definida como la transformación de tejido endometrial hacia tejido óseo y la persistencia de restos óseos de una pérdida gestacional anterior. Presentamos este caso clínico manejado en nuestra institución con una breve revisión de la literatura respectiva.
Fecha recibido: marzo I de 2010 - Fecha aceptado: junio 20 de 2010

* Instructor Asistente de Endoscopia Ginecológica, Fundación Universitaria de Ciencias de la Salud, Hospital de San José, Bogotá D.C. Colombia.
** Residente de III año, Ginecologia y Obstetricia, Facultad de Medicina, Fundación Universitaria de Ciencias de la Salud, Bogotá D.C. Colombia. 


\section{Descripción del caso}

Se trata de una mujer de 37 años remitida al servicio de endoscopia ginecológica del Hospital de San José de Bogotá, por el hallazgo de una imagen hiperrefringente en la ecografía pélvica que se ordenó como parte de estudio básico de infertilidad (Figura 1). La paciente no tiene antecedentes médicos ni quirúrgicos y dentro de su historia ginecológica trece años antes, en su única gestación, presentó aborto espontáneo durante el primer imestre sin realizarse legrado o evacuación uterina. Sus ciclos menstruales posteriores fueron normales, sin dolor ni otras alteraciones, pero sin conseguir nuevo embarazo.

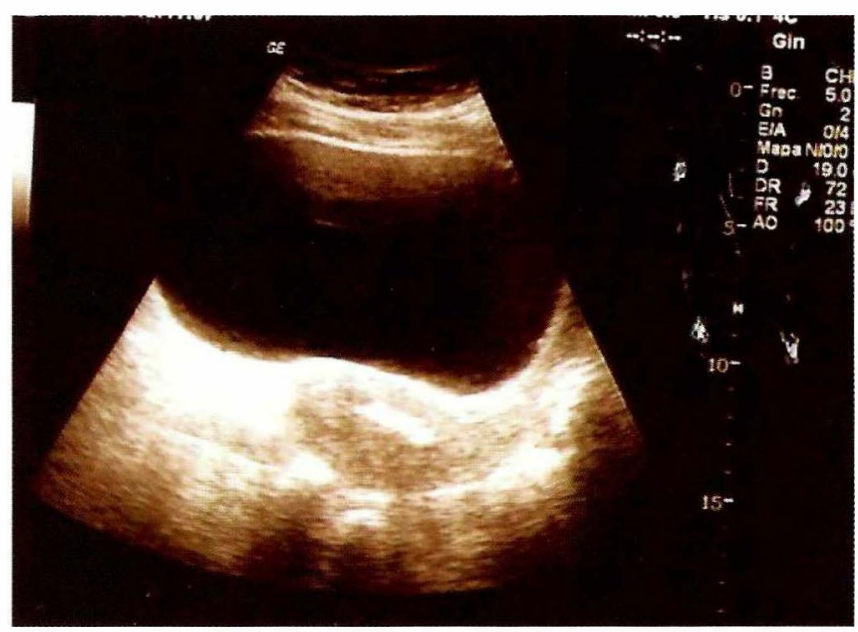

Figura I. Ecografía pélvica que revela una imagen hiperrefringente dentro de la cavidad uterina similar a un dispositivo intrauterino.

$\mathrm{Al}$ examen físico general y ginecológico no hay datos positivos por lo que se programa para histeroscopia que se lleva a cabo bajo anestesia general inhalatoria con paciente en posición de litotomía. Previa asepsia $\mathrm{y}$ antisepsia se introdujo el histeroscopio tipo Bettochi con camisa operatoria a través del cérvix con solución salina normal como medio de distensión. Dentro de la cavidad uterina se encontraron gran cantidad de fragmentos óseos que la ocupaban en su totalidad, observando también un endometrio proliferativo temprano y con los orificios tubáricos de aspecto normal (Figura 2). Utilizando pinzas de agarre se retiraron siete fragmentos óseos (Figura 3), dejando vacía la cavidaduterina sin ninguna complicación. Hubo sangrado aproximado de diez centímetros cúbicos y la duración del procedimiento fue de 30 minutos. La paciente evolucionó en forma adecuada y el reporte de anatomía patológica confirmó el diagnóstico clínico de metaplasia ósea endometrial.

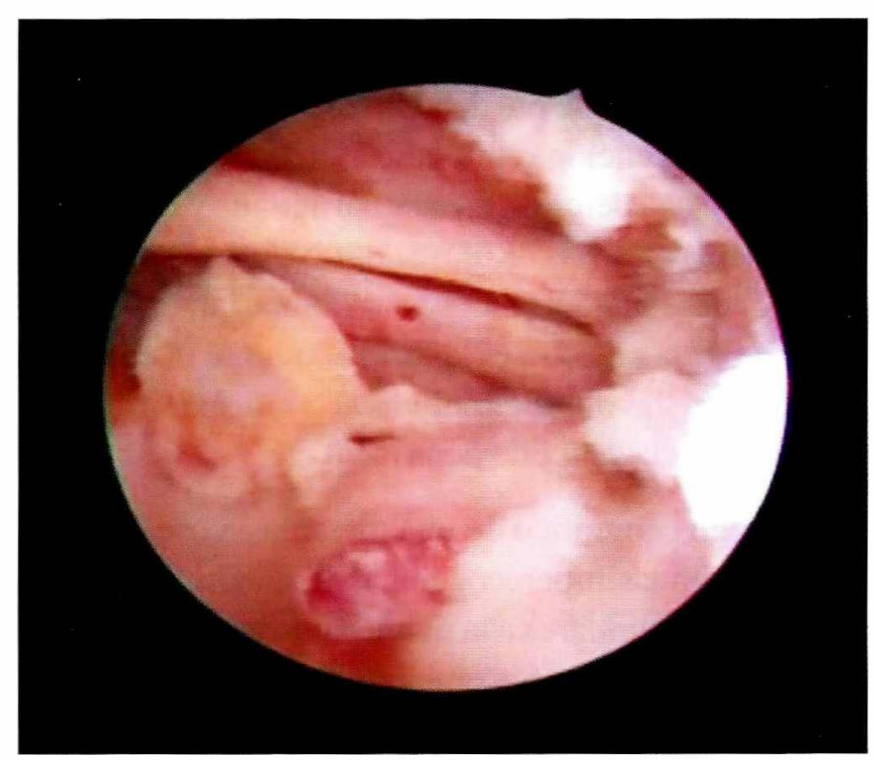

Figura 2. Imagen histeroscópica de la cavidad endometrial ocupada por múltiples fragmentos óseos.

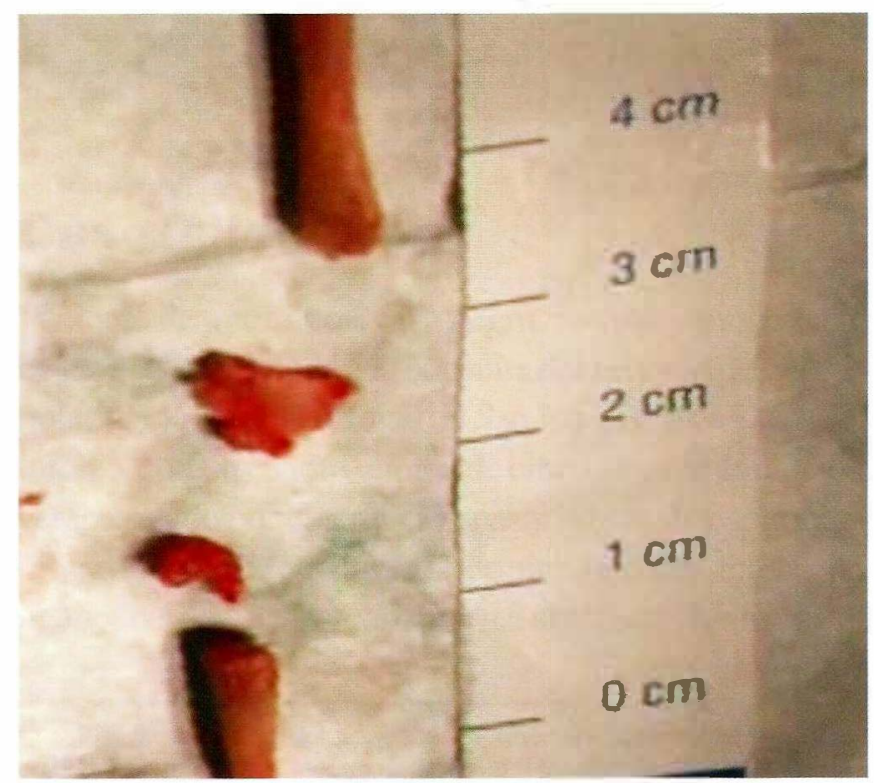

Figura 3. Vista de algunos de los fragmentos óseos después de retirados de la cavidad uterina. 


\section{Discusión}

La metaplasia ósea endometrial es una entidad de baja frecuencia con una incidencia estimada de $3: 10.000^{1} \mathrm{y}$ su principal síntoma es infertilidad secundaria en mujeres en edad reproductiva. Otras manifestaciones que suelen aquejar a estas pacientes son dolor pélvico, dismenorrea, sangrado uterino anormal o aparentes abortos. El antecedente de una pérdida gestacional previa es muy frecuente pero no un factor de riesgo obligatorio ${ }^{2}$. Hay dos teorías que pueden explicar esta patología, la primera la retención e implantación de tejidos fetales después de una pérdida gestacional antigua y la segunda una metaplasia de las células endometriales, denominada verdadera metaplasia ósea endometrial; el análisis genético de la muestra es la forma como se puede diferenciar entre las dos alternativas al confirmar o descartar la presencia de tejido de la paciente. ${ }^{3}$

El hallazgo ecográfico de una metaplasia ósea corresponde a bandas hiperecogénicas llenando la cavidad endometrial, similares a la imagen que proyecta un dispositivo intrauterino. Sin embargo, la histeroscopia es el gold estándar para la confirmación del diagnóstico mos- trando espículas y fragmentos óseos en la cavidad uterina, ${ }^{4}$ aunque la confirmación histológica es necesaria para el diagnóstico definitivo de la entidad. ${ }^{5}$ En conclusión presentamos el caso poco frecuente de una paciente con metaplasia ósea endometrial. Se recomienda la histeroscopia como herramienta fundamental no sólo para el diagnóstico sino también para el manejo definitivo.

\section{Referencias}

1. Adamson NE, Sommers SC. Endometrial ossification: report of two cases. Am J Obstet Gynecol. 1954; 67:187-90

2. Onderoglu LS, Yarali H, Gultekin M, et al. Endometrial osseus metaplasia: an evolving cause of secondary infertility. Fertil Steril. 2008;90:2013e92013 el l

3. Cayuela E, Perez-Medina T, Vilanova J, et al. True osseous metaplasia of the endometrium: the bone is not from a fetus. Fertil Steril. 2009; 91: 1293e I$1293 \mathrm{e} 4$

4. Corvalan A, Sanhueza P, Metaplasia osea endometrial con sospecha diagnostica ecográfica o histeroscopica. Rev Chil Obstet Ginecol. 2002;67:125-28

5. Shimizu M, Nakayama M.Endometrial ossification in a postmenopausal woman. J Clin Pathol. 1997;50:171-172 